\title{
Artelogie
}

Recherche sur les arts, le patrimoine et la littérature de l'Amérique latine

$9 \mid 2016$

Horizons et perspectives de la culture en Colombie (1990-2015)

\section{Identité culturelle et mémoire en Colombie contemporaine}

\author{
Obed Frausto Gatica, Nayar López Castellanos et Matthew \\ Lorenzen Martiny
}

\section{(2) OpenEdition \\ Journals}

Édition électronique

URL : http://journals.openedition.org/artelogie/278

DOI : 10.4000/artelogie.278

ISSN : 2115-6395

Éditeur

Association ESCAL

Référence électronique

Obed Frausto Gatica, Nayar López Castellanos et Matthew Lorenzen Martiny, « Identité culturelle et mémoire en Colombie contemporaine », Artelogie [En ligne], 9 | 2016, mis en ligne le 20 juin 2016, consulté le 18 novembre 2020. URL : http://journals.openedition.org/artelogie/278 ; DOI : https:// doi.org/10.4000/artelogie.278

Ce document a été généré automatiquement le 18 novembre 2020.

Association ESCAL 


\title{
Identité culturelle et mémoire en Colombie contemporaine
}

\author{
Obed Frausto Gatica, Nayar López Castellanos et Matthew \\ Lorenzen Martiny
}

\section{Introduction}

1 Les temps actuels posent de nouveaux défis dans le champ des sciences sociales. L'humanité traverse une période complexe dans laquelle le capitalisme néolibéral et déprédateur contribue à la perpétuation des guerres, à la paupérisation de millions de personnes et à la dévastation de l'environnement à partir de l'extraction accélérée de ressources stratégiques pour le développement du système et $\mathrm{du}$ mode de vie capitalistes.

2 Dans ces circonstances, le devenir historique des nations, touché par la diversité culturelle qui caractérise les peuples, les groupes ethniques et les communautés, constitue un facteur fondamental pour viabiliser des dynamiques équilibrantes dans les sociétés contemporaines. En effet, ces sujets socio-ethniques maintiennent et renouvellent une relation basée sur une conception non dichotomique entre l'homme et la nature, sur la durabilité environnementale pour les générations futures, ainsi que sur des formes d'organisation sociopolitique et économique communautaires qui peuvent être des alternatives à celles qui sont aujourd'hui hégémoniques, révélant souvent une défense des territoires et des ressources stratégiques. C'est pour ceci que nous affirmons que la diversité culturelle et la mémoire collective sont des conditions essentielles pour la continuité de l'espèce humaine.

3 Le cas colombien représente une référence dans la région latino-américaine qui offre des éléments pour la réflexion et le débat concernant la reconnaissance des droits des peuples indigènes et afro-descendants, non seulement à propos de leur identité culturelle mais aussi de leurs territoires.

4 Ce texte analyse plusieurs références conceptuelles pour encadrer le moment actuel dans lequel la Colombie se trouve dans un processus politique avec l'objectif de trouver 
la paix et de laisser derrière elle plus de sept décennies de conflit interne. Dans ce contexte, la consolidation du respect pour la diversité culturelle et le droit de créer une mémoire collective au sujet du conflit constituent deux éléments fondamentaux pour l'avenir immédiat de ce pays sud-américain.

\section{Diversité culturelle, multiculturalisme, identité}

5 Pour analyser la diversité culturelle, nous devons faire référence aux discussions philosophiques et dans les sciences sociales au sujet du multiculturalisme, qui, dans les dernières décennies, est devenu un concept central dans le débat intellectuel et dans le milieu institutionnel. Cette notion trouve ses origines dans des approches étatsuniennes qui ont cherché à promouvoir des politiques de développement et de reconnaissance des groupes ethniques face à l'oppression historique qu'ils ont vécue le long de l'histoire (TUBINO, 2003).

6 Ensuite, le concept de multiculturalisme s'est utilisé dans divers pays qui ont été d'importants récepteurs d'immigrants, comme le Canada, l'Australie, le Royaume-Uni, ou les Pays-Bas. L'innovation politique du multiculturalisme est l'idée que les droits ne doivent pas se baser exclusivement sur des droits individuels que chacun possède dans une démocratie libérale, mais qu'ils peuvent aussi se baser sur des communautés, des ethnies d'origine et leur culture, en tant que médiateurs face à l'État. Ceci serait pertinent dans, au moins, quatre types de situations. La première concerne les communautés qui habitent dans un espace géographique délimité à l'intérieur d'un État-nation. Des cas comme le Québec, le Pays Basque et le Pays de Galles sont emblématiques. La deuxième situation concerne diverses communautés qui partagent un même milieu, par exemple, dans le cas des chicanos, des hispaniques et des noirs aux Etats-Unis. La troisième situation est liée aux migrants qui ont une langue et une culture différentes à celles des pays où ils résident. La quatrième situation concerne les groupes ethniques qui ne vivent plus dans leurs territoires ancestraux et qui coexistent avec d'autres groupes ethniques. Ceci est surtout le cas des peuples indigènes d'Amérique Latine.

7 Une des caractéristiques du multiculturalisme est la reconnaissance explicite dans le domaine constitutionnel des us et coutumes des communautés, ainsi que, dans de nombreux cas, de leur autonomie politique, même si cette reconnaissance ne se traduit pas toujours dans les faits. Cependant, ceci s'oppose aux fondements de la théorie politique libérale, qui conçoit l'égalité formelle des individus, peu importe leur appartenance à une communauté ou à un groupe ethnique ou racial. Cette égalité formelle occultait un critère notoire d'exclusion et d'oppression raciale, parce qu'elle effaçait d'une certaine manière la mémoire de dépouillement et d'imposition liée à l'histoire des communautés.

8 Les États qui ont adopté le multiculturalisme tentent de réconcilier le principe de l'égalité formelle des individus et le principe de la différence culturelle des communautés. Mais ceci représente justement le dilemme, qui est exprimé par diverses opinions qui défendent la notion de multiculturalisme, qui, au contraire, cherchent à maintenir les valeurs juridiques de la théorie politique libérale, ou qui proposent la dissolution des théories libérales.

9 Un des auteurs libéraux qui critiquent ouvertement la reconnaissance juridique de la diversité culturelle est Giovanni Sartori, qui argumente que cette reconnaissance 
favorise la ghettoïsation et met en danger les fondements de la démocratie libérale (SARTORI, 2001). Charles Taylor, de son côté, soutient que le multiculturalisme implique que des cultures luttent pour être reconnues face à d'autres cultures, et que cette reconnaissance signifie une revendication et une valorisation de ces premières cultures, qui cherchent de l'autonomie (TAYLOR, 1979). L'État devrait donc accorder une reconnaissance juridique aux différentes cultures, et on devrait effectivement aller au-delà du libéralisme classique. De son côté, Michaël Walzer considère qu'il n'y aurait pas de raison pour aller aussi loin, parce qu'il serait possible d'accorder une reconnaissance aux différents groupes dans le cadre de l'État démocratique libéral (WALZER, 1983).

10 C'est ainsi qu'un débat s'est initié entre des positions libérales et communautariennes. La théorie libérale a toujours affirmé que c'est dans la reconnaissance des droits individuels universels que les minorités ethniques seraient protégées. La protection des minorités serait indirecte parce que, malgré le manque d'une mention spécifique qui les concerne, la défense des droits individuels serait la clé pour inclure tous les groupes.

11 Dans le débat entre libéraux et communautariens il y a eu un éventail de propositions, et, malgré le fait qu'elles s'identifient comme appartenant à l'une ou l'autre approche, elles tentent souvent de combiner plusieurs idées des deux côtés. Ce qui est vrai c'est que chaque proposition contient des fondements différents. En général, les libéraux considèrent que le respect mutuel entre les différents individus doit prévaloir, malgré leurs conceptions différentes ; que le principe de non ingérence, qui implique le respect et la non intrusion dans la vie des autres, est fondamental ; et qu'il doit y avoir une diversification des domaines (politique, social, culturel, etc.) de la vie sociale (RAWLS, 1985).

12 À partir des années 1980, des critiques de la théorie libérale ont surgi, particulièrement en ce qui concerne sa vision atomiste des individus. Des auteurs tels que Taylor, MacIntyre et Sandel ont argumenté que la reconnaissance des droits communautaires et collectifs était nécessaire (TAYLOR, 1979; MACINTYRE, 1981; SANDEL, 1982). La position communautarienne constate que la société est fragmentée et affirme qu'il est nécessaire de reconnaitre les différentes communautés qui existent en son sein. Cette position argumente que la communauté est le lieu où l'identité des individus est formée, et que sans communauté morale il n'y aurait pas d'individus moraux.

13 Malgré leurs différences, les deux positions tendent à accepter l'importance des valeurs morales de reconnaissance mutuelle afin de garantir une harmonie entre les individus. Cependant, la morale est une lutte de volonté de puissance ; en conséquence, la raison pratique devrait faire face au principe d'oppression et de domination entre les cultures. Ainsi, l'identité ne se constitue pas seulement dans la communauté d'appartenance de chaque individu; elle est aussi disputée et il existe une lutte pour la reconnaissance du ressentiment lié aux siècles d'extermination, de soumission culturelle et de perte de la mémoire. De plus, comme l'affirme Briones, la race et l'ethnie ne sont pas des catégories établies; les identités varient dans les divers contextes sociaux selon l'économie et la politique de la production d'hégémonies culturelles où il existe sans aucun doute un réseau social d'exclusions et d'inclusions, et un jeu ambivalent entre récupération et perte de la mémoire (BRIONES, 1998).

14 Face à ce contexte, selon Fernández, il serait nécessaire de comprendre l'identité culturelle latino-américaine à partir d'une position commune, respectant les 
différences mais en termes d'une égalité intégrale sans centres de domination ou d'hégémonie culturelle. Dans ses propres mots :

Son essence se trouve dans le dialogue interculturel avec l'autre, en conditions d'égalité, sans exclusions. Celui qui le porte est l'homme concret, où l'universel y le singulier se conjuguent à travers la dialectique. Il se manifeste dans des espaces géographiques spécifiques et des temps déterminés. Il se construit à partir des activités et la volonté des hommes dans le processus historique. (FERNÁNDEZ, 2011 : 95)

La conformation politique, économique et culturelle des nations latino-américaines et caribéennes a été marquée par l'irruption des puissances européennes qui, durant trois siècles de colonisation, ont ébranlé les structures indigènes pour imposer des modèles culturels, linguistiques et religieux appartenant à ce qui est nommé génériquement la " civilisation occidentale ». Les structures indigènes ont été réduites aux dénommées " républiques d'indiens ", qui payaient des tributs aux conquérants et étaient soumises à des conditions extrêmes d'exploitation et de spoliation, leur niant l'exercice de droits politiques, économiques ou sociaux.

$17 \mathrm{Au}$ début du xixe siècle, les élites créoles qui ne supportaient plus le fait d'être subordonnées aux monarchies européennes en décadence ont impulsé l'indépendance des pays latino-américains. Ces élites cherchaient à s'approprier des entités politiques nouvelles sans modifier les structures d'exploitation, de racisme et de contrôle de la population qui ont existé sous la domination coloniale.

18 Ainsi, durant ce siècle, des États oligarchiques se sont consolidés, conservant un système de colonialisme interne, dépouillant de nombreuses manières aux communautés indigènes des territoires où ils ont survécu durant la période coloniale. À ce sujet, Florescano remarque, en ce qui concerne le cas mexicain, que :

[...] les indigènes n'ont pas seulement perdu le fondement légal de la propriété communale ; ils se sont aussi transformés en parias politiques, parce que ni l'État ni les groupes qui se disputaient le contrôle de la nation n'ont défendu leur cause ou proposé des procédures qui auraient permis leur intégration dans le projet national. Au contraire, nous pouvons dire que la consigne qui s'est imposée est celle de s'emparer des terres indigènes, de détruire les institutions qui permettaient la cohésion des identités ethniques, et de combattre les traditions, les cultures et les valeurs indigènes. (FLORESCANO, $1997: 431$ )

19 Durant les premières décennies du xxe siècle, les vieilles oligarchies ont été supplantées par les bourgeoisies nationales qui ont structuré des systèmes politiques basés, dans le meilleur des cas, sur la démocratie représentative. La construction de cette nouvelle hégémonie s'est produite suivant la dynamique du colonialisme interne, un processus à partir duquel les populations indigènes n'ont pas seulement été exclues de tout droit politique mais ont été aussi conquises nouvellement à partir de leur « incorporation » violente au projet national.

Il faut indiquer que le concept de colonialisme interne a été développé par Rodolfo Stavenhagen et Pablo González Casanova dans les années 1960. Ce concept tente d'expliquer comment les structures de pouvoir dans les nations "indépendantes" d'Amérique Latine conservent les dynamiques du colonialisme européen, à partir de la supposée supériorité et civilité occidentale face à des indigènes arriérés et sauvages, ce qui justifiait leur contrôle, l'imposition d'une éducation étrangère et leur exploitation.

21 Ainsi, les résistances et rébellions indigènes forment partie de ce complexe processus de construction des nationalités, dans lesquelles l'origine préhispanique ne représente 
qu'un imaginaire d'utilité discursif, et même folklorique, pour donner un contenu à ces nouvelles identités et un point d'ancrage culturel. Historiquement, le contenu de ces résistances s'est limité à s'opposer aux structures extrêmes d'exploitation et d'exclusion, et au dépouillement de leurs territoires. Aucun projet intégral de libération n'existait, ou tout au moins aucun ne s'est consolidé, et aucune conception concrète concernant des transformations intégrales dans les domaines politique, économique et social ne s'est produite.

Ce n'est que dans la période contemporaine que des programmes plus élaborés se sont profilés et que les luttes indigènes se sont reconfigurées à partir de postulats tels que l'autonomie, la libre détermination et l'exigence de pouvoir exercer des droits politiques, économiques, sociaux, culturels et territoriaux, non seulement en tant que citoyens mais aussi en tant que communautés. Les efforts d'organisation et de création de ces nouveaux paramètres de lutte impliquent un virement radical dans les résistances indigènes, dans lesquelles l'aspect politique, en particulier l'exigence du droit à s'autogouverner, est un élément partagé par la majorité des mouvements qui s'opposent aux oligarchies et aux États à leur service.

Jouent un rôle essentiel, dans ce processus de reconfiguration des résistances indigènes, les idées formulées à partir des années 1970 par une génération d'intellectuels et d'académiques qui, depuis les champs de l'anthropologie, la sociologie, l'histoire et les sciences sociales en général, ont modifié l'analyse et l'interprétation des peuples indigènes, construisant, parallèlement aux mouvements et aux apports de ces peuples, une nouvelle vision concernant leurs luttes et les processus d'exploitation, de racisme et de discrimination auxquels sont soumis les communautés indigènes.

\section{Colombie : le changement constitutionnel de 1991}

Durant les dernières années de la décennie de 1980 et durant la décennie de 1990, diverses constitutions des pays d'Amérique Latine ont connu l'inclusion de la diversité culturelle, incorporant aux structures institutionnelles libérales des droits collectifs ethniques, principalement en ce qui concerne l' «indienneté » (ASSIES, 2000 ; COTT, 2000). Quelques exemples incluent les constitutions du Nicaragua en 1987, du Brésil en 1988, de la Bolivie en 1994 et de l'Équateur en 1998. En Colombie, un nouveau processus constituant a culminé avec la rédaction d'une nouvelle constitution en 1991, qui présentait comme nouveauté la possibilité de créer des entités territoriales indigènes, pouvant réunir différentes communautés sous une même autorité indigène. Les communautés indigènes ont exigé la restitution des terres de la Sierra Nevada, qui avaient été saisies historiquement par les blancs.

Les communautés indigènes ont commencé à se politiser dans les années 1970 en raison de l'exclusion de leur condition de culture indigène à partir de l'idée du métissage, qui effaçait la mémoire et les origines de leur culture lors de la formation d'une identité nationale unitaire. La rupture peut s'expliquer en partie par l'échec, dans cette décennie, du modèle économique basé sur la notion de citoyenneté avec des droits économiques individuels. Quelques années plus tard, une crise économique régionale a secoué l'Amérique Latine, liée à l'incapacité des pays de rembourser leurs dettes extérieures et aggravée par les conditions imposées par les institutions financières internationales, impliquant des politiques radicales de libéralisation commerciale, de 
privatisation et d'austérité, limitant les capacités des États de satisfaire les demandes économiques et sociales des populations.

Ainsi, les gouvernements des pays latino-américains ont déraciné l'État développementaliste et se sont pliés au "Consensus de Washington", un modèle politico-économique impliquant le soutient inconditionnel à l'initiative privée, la discipline fiscale, la libéralisation commerciale, et la réduction du rôle de l'État dans la direction du développement économique. Dans son œuvre Capitalisme et liberté, publié en 1962, Milton Friedman affirmait que « à moins que nous atteignions ou conservions la liberté économique, toutes les autres libertés - civiles ou politiques - se dissipent... L'enseignement est clair : le libéralisme peut ne pas être une condition suffisante, mais certainement il est une condition nécessaire pour atteindre la liberté globale. » (Cité dans MERQUIOR, 1993 : 170).

Le libre-échange s'est transformé dans le principal outil des capitaux transnationaux pour assurer leur reproduction massive. C'est ainsi que les principales puissances mondiales, avec les Etats-Unis en tête, ont impulsé des accords de libre-échange avec des pays sous-développés dans des conditions complètement inégales. Il s'agit d'un colonialisme moderne, réalisé sans la nécessité d'occuper des territoires, et facilité par la globalisation néolibérale que nous caractérisons, suivant la conception de Valdés, comme un Système de Domination Multiple (SDM). Celui-ci est constitué de cinq axes fondamentaux : l'exploitation économique et l'exclusion sociale ; l'oppression politique dans le cadre de la démocratie formelle ; la discrimination socioculturelle ; l'aliénation médiatique et culturelle; et la déprédation écologique. Les projets de libre-échange constituent un élément additionnel fondamental, fonctionnant comme des entités supranationales. De plus, Valdés affirme qu'avec cette catégorie

[...] nous pouvons visualiser l'ensemble des formes de domination et d'assujettissement, dont certaines sont restées invisibilisées pour la pensée critique, et nous pouvons encourager le rapprochement entre diverses demandes et pratiques émancipatrices qui semblent être aujourd'hui opposées ou non articulées, et éviter de cette manière des réductionnismes, anciens ou nouveaux, liés à la prédétermination abstraite d'acteurs sociaux à qui sont attribués a priori des tâches libératrices messianiques. (VALDÉs, 2009 : 14)

Pour Perry Anderson, le néolibéralisme s'est constitué comme un vrai mouvement idéologique à niveau international, impulsé essentiellement par la droite, et ayant généré

[...] un degré de succès probablement jamais imaginé par ses fondateurs, disséminant l'idée simple qu'il n'y a pas d'alternatives à ses principes et que tous, partisans ou opposants, doivent s'adapter à ses normes. Probablement aucun savoir conventionnel n'a réussit à atteindre une prédominance si large depuis le début du siècle comme le cas du néolibéralisme. (ANDERSON, 1995 : 23)

À partir d'une vision globale, Kaplan argumente que l'État-nation se trouve dans un processus de dissolution sous l'effet d'un ensemble d'acteurs politiques, économiques et militaires, auxquels s'ajoutent aujourd'hui un nombre croissant d'acteurs, y compris les entreprises transnationales; les organisations internationales (les Nations Unies et ses agences, le Fonds Monétaire International, la Banque Mondial, la Banque Interaméricaine de Développement, l'Organisation Mondiale du Commerce) et les organismes régionaux; les mouvements et organisations non gouvernementales multi et transnationales (ethniques, religieuses, idéologiques, féministes, de jeunesse, politiques, écologistes, défenseuses des droits humains, etc.) ; les grands spéculateurs 
financiers; les agences de notation financière (Standard \& Poor's, Moody's); des organisations criminelles, subversives et terroristes ; ou encore des groupes nomades et apatrides (membres des élites économiques, migrants économiques, réfugiés politiques, parmi d'autres) (KAPLAN, $2002: 168$ ).

Dans ce contexte national et international, un ensemble consolidé de forces politiques est apparu en Colombie exigeant l'application de la Loi 89 de 1890, qui déclarait que les terres communautaires, appelées resguardos, étaient inaliénables. Cette loi représentait le cadre juridique qui a permis au mouvement indigène d'obtenir une reconnaissance dans la Constitution de 1991 (GROS, 2012).

De manière parallèle, la crise de légitimité des successifs gouvernements colombiens s'est approfondie. Ceci s'explique par la croissance du narcotrafic et de la violence dans tout le pays, par la crise de crédibilité des partis politiques, par l'accroissement de la corruption au sein de l'élite politique, et par la privatisation continue de divers secteurs de l'État et l'élimination de politiques publiques, qui ont aggravé les disparités entre les secteurs les plus pauvres de la société et les secteurs les plus nantis. L'État cherchait d'urgence rénover le discours pour trouver une source de légitimité qui lui permettrait sa reconstruction. C'est ainsi que la narrative de la pluralité ethnique s'est peu à peu construite.

La Constitution de 1991 est devenue une sorte de mythe politique fondateur (MEJÍA, 1998), produit d'un esprit réformateur et d'une réconciliation de différents secteurs de la société qui cherchaient un renouvellement pour confronter les grands problèmes nationaux. L'Assemblée Nationale Constituante a été perçue comme un espace démocratique et pluraliste où différents secteurs politiques et culturels pouvaient se rencontrer et dialoguer. Les sujets qui ont été analysés dans l'Assemblée étaient d'une grande importance pour l'imaginaire social, et incluaient les droits humains, la paix, la participation de la société civile, la démilitarisation et la diversité culturelle (SAFFORD et PALACIOS, 2002). Le processus constituant a fait participer un grand nombre de secteurs sociaux et a produit une transformation des acteurs politiques les plus centrés sur la catégorie de diversité.

Cependant, cette rénovation du discours a trouvé des défis intéressants concernant le multiculturalisme. Par exemple, deux problématiques incluent la difficulté d'accoupler l'Etat national avec la diversité d'identités, et la complexité de réconcilier les droits individuels et les droits culturels. C'est important de remarquer que la Constitution donne plus de poids aux droits des blancs et des métisses qu'à ceux d'autres groupes. Ceci est évident par le fait que, dans le contexte néolibéral, les droits individuels des propriétaires, basés essentiellement sur la capacité financière, deviennent corporatifs, se répandant à tous les domaines de la vie et détériorant les droits communautaires. Même si des droits communautaires des groupes indigènes ont été reconnus dans la Constitution, cette reconnaissance n'a pas bénéficié aux personnes noires, malgré le fait que la population indigène représente à peine $2 \%$ de la population totale de la Colombie et la population noire environ $20 \%$ de la population. 


\section{Tensions entre les droits individuels et les droits collectifs}

Sans aucun doute, une des tensions les plus significatives de la Constitution concerne les limites juridiques entre les droits individuels et les droits communautaires. La Constitution reconnait le droit collectif des communautés indigènes à un territoire ainsi qu'un ensemble de dispositions juridiques complémentaires y compris le droit des communautés à s'autogouverner. Selon la Constitution, les indigènes sont des groupes qui habitent dans une région géographique depuis le moment de la conquête jusqu'au présent, et qui maintiennent en quelque sorte leurs traditions sociales, économiques, politiques et culturelles. Dans l'article 7 de la Constitution, l'État reconnaît la diversité culturelle et ethnique de la nation. La Constitution reconnaît aussi le droit à s'autogouverner à partir des us et coutumes, le droit à la protection de leur environnement, et deux sénateurs indigènes sont mêmes élus à niveau national.

Une des tensions entre les droits individuels et les droits communautaires s'explique par la prédominance juridique des droits individuels, par-dessus des droits collectifs. Le problème est que l'État est considéré comme une institution neutre et objective qui trouve des équilibres entre ces deux types de droits. Cependant, l'État est immergé dans un système de valeurs, parce qu'il privilégie une langue, certaines valeurs et coutumes, et même certains rituels par-dessus d'autres. Tout cela se fait malgré la reconnaissance de la diversité culturelle dans le domaine juridique, la lutte pour la reconnaissance des différences culturelles, et les luttes pour récupérer et protéger les identités communautaires. Ceci suppose une dispute axiologique entre les valeurs individuelles et les valeurs communautaires, qui se traduit dans des cas concrets, par exemple lorsque certaines personnes qui font partie des communautés transgressent les us et coutumes. Les punitions que les communautés imposent à ces personnes pourraient transgresser les droits octroyés aux individus par l'État. L'État se trouve, dans ces circonstances, dans le dilemme de respecter les us et coutumes des communautés ou de respecter le droit individuel de chaque individu. Dans ce dilemme, le droit à la diversité culturelle est celui qui perd parce qu'il y a un biais culturel qui se traduit par le fait que l'État intervient pour protéger les formes de vie et les intérêts des citoyens.

La reconnaissance juridique de la diversité culturelle a provoqué le surgissement de demandes de reconnaissance de la part de plusieurs ethnies. Cependant, l'État a confronté des problèmes pour déterminer comment cataloguer certaines communautés et quels droits leurs octroyer. Il y a des groupes qui se reconnaissent à eux-mêmes comme indigènes, et défendent leur identité indigène, mais qui ne possèdent pas les caractéristiques générales pour être considérés comme indigènes, notamment parce qu'ils n'ont pas de langue ou d'us et coutumes distincts. Un cas très clair est celui des mokana (BORDA, 2009), ce qui rend très clair la difficulté de définir des identités, puisque les identités sont constamment en dispute, dans un jeu de reconnaissance et méconnaissance. Cependant, l'État, qui a une logique implicite de colonialisme, est celui qui décide si une communauté peut être cataloguée ou non comme un groupe indigène. $\mathrm{Ce}$ jeu de reconnaissance et méconnaissance est inéquitable parce que les communautés doivent s'efforcer énormément pour obtenir une reconnaissance et c'est l'État qui octroi ou non cette reconnaissance. est important de présenter quelques réflexions sur la notion de négritude. Peter Wade a 
réalisé des études où il montre comment les indigènes et les noirs sont inscrits dans un processus historique qui structure les altérités et qui suppose des ruptures et des continuités. Wade montre que durant la période coloniale des asymétries se sont formées dans l'espace social, vis-à-vis les indigènes et les noirs (WADE, 1999). Nina de Friedemann, de son côté, considère qu'en raison du manque d'études sur les noirs, contrairement au cas des indigènes, il est possible d'affirmer qu'il y a une invisibilisation de ces premiers (FRIEDEMANN, 1984).

La Constitution colombienne reconnaît la diversité, mais surtout concernant le cas et les formes de vie indigènes, accordant des droits communautaires et l'autodétermination à partir d'un territoire occupé avant la conquête. Cependant, la communauté noire ne bénéficie pas d'une reconnaissance pareille, malgré le fait qu'elle représente environ $20 \%$ de la population. Les noirs sont les descendants des esclaves africains qui ont été amenés en Colombie pour être exploités dans les latifundios. La conception de la diversité culturelle concernant les indigènes suppose que ces peuples ont été dépouillés des territoires qu'ils possédaient avant la conquête. Le problème est que les esclaves n'ont jamais eu de territoire et que leur arrivé dans le pays a été le produit de l'oppression et de l'esclavage.

Plusieurs auteurs ont utilisé le modèle de l'ethnicité indigène pour faire référence aux communautés noires (AGUDELO, 2005). Wade considère que la législation qui reconnaît les communautés noires comme un groupe ethnique avec des droits territoriaux et culturels suit le modèle indigène. En Colombie, comme le suggère un texte de Ng'Weno, les populations noires qui habitent dans la région du Pacifique ont été constituées légalement suivant le paradigme de l'ethnicisation (NG'WENO, 2007). Cependant, d'autres afro-descendants en dehors de cette région ne trouvent pas un chemin facile pour cette reconnaissance. Restrepo affirme que l'utilisation de la notion d'ethnicité dans le cas des afro-colombiens est un fait relativement nouveau et est lié à la catégorie de sociétés primitives (RESTREPO, 2002, 2007). Dans la Constitution, leurs droits politiques sont associés à un jeu dichotomique concernant le primitif et le moderne, ce qui veut dire que la Constitution a pratiquement effacé les noirs comme un groupe ethnique qui peut lutter pour la reconnaissance de ses communautés dans le cadre de la diversité culturelle. C'est ainsi que le cadre juridique de la diversité ethnique est très inflexible par rapport à l'énorme complexité des identités colombiennes.

\section{Mémoire}

La mémoire est une partie essentielle de l'identité d'un espace collectif, intégré par des individus qui partagent des dynamiques et des faits qui construisent leur histoire et leur appartenance à un espace déterminé. Néanmoins, la mémoire peut être aussi restreinte et mutilée, particulièrement dans des contextes conflictuels ou violents; des situations dans lesquelles la vision du vainqueur tente d'être imposée et tout acte criminel qui a permis la condition de vainqueur tente d'être effacée.

Selon Ana Graciela Galindo, faisant référence à un texte de Pilar Riaño,

[...] les mémoires sont multiples, avec de constants et de longs conflits, des désaccords et des sentiments, dans lesquels le gens trouvent la possibilité de s'impliquer et de faire partie d'un collectif. La mémoire serait donc perçue comme une pratique culturelle qui met en œuvre des connaissances, des actions et des expériences permettant de nous situer et de discerner qui nous sommes. (GALINDO, 2011:52) 
41 Une des expressions les plus concrètes de la défense de la mémoire à partir des conflits armés comme celui de la Colombie est la création d'une Commission de la Vérité qui relève le défi d'enquêter sur les violations systématiques des droits humains dans le cadre d'un conflit armé, en cherchant la vérité, l'identification des responsables intellectuels et matériels, ainsi que la possible réparation du préjudice. La Commission de la Vérité doit garantir une connaissance sans restriction des faits et des évènements qui ont eu lieu dans un conflit touchant une population civile non impliquée dans le combat, en dévoilant la dimension des crimes comme le pilier fondamental d'une vraie cicatrisation collective.

Ignacio Ramonet constate que :

[...] la reconstruction de l'identité sociale est un travail collectif qui suppose la possibilité d'élaborer un récit vérace des crimes commis par l'autoritarisme... Pour que la mémoire ne se dégrade pas, il est nécessaire de la mettre en pratique en considérant le présent et l'avenir. Pour mettre fin à l'impunité et éliminer la possibilité que les crimes et génocides se répètent, il est indispensable de sauver la mémoire à propos des luttes contre l'injustice sociale, pour la souveraineté, pour les libertés, et pour les droits humains. (RAMONET, $2016: 2$ )

Dans le contexte du conflit en Colombie, la mémoire comme signe d'identité demande une intervention majeure à travers la Commission de la Vérité en concordance avec les Accords de paix entre le gouvernement et les Forces armées révolutionnaires de Colombie (FARC), puisqu'il s'agit d'offrir une réponse éthique à une société abattue par des décennies de souffrance collective liée à une violence qui a diverses expressions, non seulement la guerre entre deux factions, mais aussi la violence des groupes paramilitaires financés par l'État, des mafias du crime organisé et de la délinquance commune.

Dans ce contexte, il est indispensable de parvenir à créer une mémoire commune, collective et inclusive qui, reconnaissant les différences de pensée et les histoires autour du conflit, puisse générer une culture et une identité liées au respect sans restrictions des droits humains et au combat contre l'impunité et contre la corruption systématique, qui font partie des structures qui ont permis les crimes contre l'humanité subis par la société colombienne durant six décennies.

Selon Fernando Arellano Ortiz,

[...] Les sociétés déstructurées comme la colombienne, qui ont subit durant des années les tourments de la guerre et de la violence endémique, ont besoin de la réalisation d'un deuil social et collectif pour faire face à la douleur et à la souffrance, ce qui peut être accompli à partir de la réélaboration du sens de la mémoire historique face à un avenir différent, impliquant les garanties de non répétition des faits atroces commis et subis dans le passé. (ARELLANO, 2016)

De son côté, Elsa Blair Trujillo note que :

[...] au besoin social (et dans l'aspect moral) des victimes d'évoquer pour guérir, et au besoin des sociétés de se rappeler (et/ou d'oublier) pour se réconcilier, s'ajoutent le besoin de comprendre comment ces expériences traumatiques compromettent non seulement le passé, en restant fixées dans la mémoire des sociétés, mais aussi l'avenir, parce qu'elles constituent la matière première de la mémoire collective de la société. (TRUJILLO, $2002: 15$ )

De cette manière, la construction d'une mémoire collective en Colombie constitue une action fondamentale pour que le processus de paix arrive à bon terme. La connaissance de la vérité de la part des victimes du conflit, les procès juridiques contre les 
responsables et les pénalités exemplaires deviennent un pont pour se frayer un chemin vers une nouvelle étape dans la vie de cette société sud-américaine.

\section{Conclusion}

La lutte d'identités dans le jeu de reconnaissance et méconnaissance a été un processus constant dans le développement historique d'Amérique Latine, notamment en Colombie. Dans la logique des hégémonies, l'actualité des nations latino-américaines a été touchée par le fardeau historique et symbolique de la conquête et la colonisation. Ainsi, il y a des auteurs qui considèrent qu'il existe encore un colonialisme interne incrusté dans les pays latino-américains, comme un héritage du passé que touche la quotidienneté ainsi que les diverses expressions de la vie sociale. Les tensions se propagent avec la violence et le conflit dans lesquels il y a un processus d'anéantissement et de méconnaissance symbolique d'autrui. Dans un contexte de discorde et d'affrontement, la mémoire joue un rôle fondamental pour atteindre une re-signification du sens de la réconciliation, de la récupération et de la justice nécessaire pour la réintégration des diverses factions en conflit. Quant à l'application du multiculturalisme dans les contextes latino-américains et la récupération de la mémoire après une période de violence ex trême, comme en Colombie, les défis à relever sont encore nombreux.

\section{BIBLIOGRAPHIE}

AGUDELO C.E., (2005). Retos del multiculturalismo en Colombia : política y poblaciones negras.

Medellín : Editorial La Carreta.

ANDERSON P. (1995). "Balanço do neoliberalismo". Pos-neoliberalismo : as políticas sociais e o

Estado democrático, Sader, E. (Ed.), Sao Paulo : Paz e Terra, pp. 9-23.

ARELLANO ORTIZ F. (2016). "Memoria histórica como identidad social colectiva y eje político transformador en el proceso de paz colombiano”. [en ligne : http://www.cronicon.net/paginas/ edi...]

ASSIES W. (2000). "Indigenous people and reform of the state in Latin America". In The challenge of diversity : indigenous peoples and reform of the state in Latin America, Assies, W, Van der Harr. G. et Hoekeman, A. (Eds.), Amsterdam : Thela, pp. 3-21.

BORDA C. (2009). "Diversidad étnica y la reconstrucción de identidades : el grupo Mokana en el departamento del Atlántico, Colombia". European Review of Latin American and Caribbean Studies, 86, pp. 39-57.

BRIONES C. (1998). La alteridad del "Cuarto Mundo". Una deconstrucción antropológica de la diferencia. Buenos Aires : Ediciones del sol.

COTT V.D. (2000). The friendly liquidation of the past. Pittsburgh : University of Pittsburgh Press. 
FERNÁNDEZ D.F. (2011). “América Latina. Diversidad y cultura”. América Latina en tiempos de bicentenario, Pérez Cruz, F.J. (Coord.), La Havane : Editorial de Ciencias Sociales, pp.70-95. FLORESCANO E. (1997). Etnia, Estado y Nación. Mexico : Taurus.

FRIEDEMANN N.S. (1984). "Estudios de negros en la antropología colombiana : presencia e invisibilidad". Un siglo de investigación social : antropología en Colombia, Arocha J. et Friedemann, N.S. (Eds.), Bogotá : Etno, pp. 507-572.

GALINDO SALAZAR A.G. (2011). “Las tensiones de la memoria”. Esfera, 1(2), pp. 47-57.

GROS C. (2012). Políticas de la etnicidad : identidad, estado y modernidad. Bogotá : ICAHN.

KAPLAN M. (2002). “El sistema internacional : límites, paradojas y posibilidades”. Globalidad y conflicto. Estados Unidos y la crisis de septiembre, Valdés, J.L. et al. (Eds.), Mexico : CISAN-IIJUNAM, pp. 163-183.

MACINTYRE A. (1981). After Virtue. Londres : Duckworth.

MEJÍA QUINTANA O. (1998). "Participación ciudadana : del mito a la realidad. Hacia una reinterpretación consensual-discursiva de la Constitución de 1991". Convergencia entre ética y política, Hoyos Vásquez G. et Uribe, A. (Eds.), Bogotá : Siglo del Hombre, pp. 145-163. MERQUIOR J.G. (1993). Liberalismo viejo y nuevo. Mexico : Fondo de Cultura Económica. MONTES MONTOYA A. (2015). «Changements juridiques dans un contexte multiculturel : le Noir en Colombie, un regard philosophique ». Les Amériques des Indépendances à nos jours. Des constitutions aux démocraties, García, J.R. (Ed.), Paris : Editions Fondation de la Maison des Sciences de l'Homme.

MONTES MONTOYA A. (2015) La Représentation du Noir dans la Colombie contemporaine : essai sur le cas de Carthagène des Indes. Paris : Collection Recherches Amériques Latines, L'Harmattan.

NG'WENO B. (2007). “Can Ethnicity Replace Race ? Afro-Colombians, Indigeneity and the Colombian Multicultural State ". Journal of Latin American and Caribbean Anthropology, 12(2), pp. 414-440. PARDO M. (2002). “Entre la autonomía y la institucionalización : dilemas del movimiento negro colombiano". The Journal of Latin American Anthropology, 7(2), pp. 60-85.

RAMONET I. (2016). “Guerras, memoria, justicia”. Le Monde diplomatique en español, 245(mars), pp. $1-2$.

RAWLS J. (1985). “Justice as Fairness : Political not Metaphysical”. Philosophy and Public Affairs, XIX(3), pp. 233-251.

RESTREPO E. (2002). "Políticas de la alteridad : etnicización de "comunidad negra” en el Pacífico sur colombiano". Journal of Latin American Anthropology, 7(2), pp. 34-59.

RESTEPO E. (2007). "El "giro al multiculturalismo" desde un encuadro afro-indígena". Journal of Latin American and Caribbean Anthropology, 12(2), pp. 475-486.

RIAÑO ALCALÁ P. (2006). “Introducción”. Jóvenes, memoria y violencia en Medellín : una antropología del recuerdo y el olvido (pp. xxix-lx), Riaño Alcalá, P. (Ed.), Medellín : Universidad de Antioquía, Instituto Colombiano de Antropología e Historia.

SAFFORD F. et PALACIOS, M. (2002). Colombia : fragmented land, divided society. New York et Oxford : Oxford University Press.

SANDEL M. (1982). Liberalism and the Limits of Justice. Cambridge : Cambridge University Press. 
SARTORI G. (2001). La sociedad multiétnica. Pluralismo, multiculturalismo y extranjeros. Madrid :

Taurus.

TAYLOR C. (1979). Hegel and Modern Society. Cambridge : Cambridge University Press.

TRUjILlO E.B. (2002). "Memoria y narrativa : la puesta del dolor en la escena pública”. Estudios

Políticos, 21, pp. 9-28

TUBINO F. (2003). “Interculturalizando el multiculturalismo”. Interculturael. Balance y

perspectivas. [http://www.cidob.org/media2/publica...]

VALDÉS GUITÉRREZ G. (2009). Posneoliberalismo y movimientos antisistémicos. La Havane : Editorial

de Ciencias Sociales.

WADE P. (1999). “The Guardians of Power : Biodiversity and Multiculturality in Colombia”. The

Anthropology of Power. Empowerment and Disempowerment in Changing Structures, Cheater, A. (Ed.), Londres et New York : Routledge, pp. 73-87.

WALZER M. (1983). Spheres of Justice. New York : Basic Books.

\section{RÉSUMÉS}

Dans cet article nous révisons le défi du multiculturalisme et de la diversité culturelle dans le contexte latino-américain, analysant le cas concret de la Colombie. Nous tentons de lier la notion de diversité culturelle à l'idée de mémoire. La Constitution colombienne adoptée en 1991 a cherché à faire le pont entre ces deux concepts; toutefois, nous estimons que les défis sont encore très grands pour cette nation andine, considérant en particulier les catégories de négritude et d'identité culturelle. Nous notons aussi les tensions qui existent dans la Constitution entre les droits individuels et les droits collectifs, et nous montrons les difficultés qui existent pour la diversité culturelle face à la percée des politiques néolibérales.

En este artículo revisamos el desafío del multiculturalismo y la diversidad cultural en el contexto latinoamericano, en particular en Colombia. Intentamos vincular la noción de diversidad cultural con la idea de memoria. La constitución política colombiana de 1991 intenta hacer el puente entre estas dos nociones; sin embargo, vemos el desafío que presenta ante la realidad colombiana principalmente con la categoría de negritud y de identidad cultural. Asimismo, hacemos notar las tensiones que existen en la constitución entre los derechos individuales en relación con los colectivos y además mostramos las dificultades que existen en la idea de diversidad cultural ante la avanzada de las políticas neoliberales.

\section{INDEX}

Palabras claves : multiculturalismo, diversidad cultural, memoria, Colombia

Mots-clés : multiculturalisme, diversité culturelle, mémoire, Colombie

\section{AUTEURS}

\section{OBED FRAUSTO GATICA}

Candidat doctoral en philosophie de la science, Universidad Nacional Autónoma de México, Professeur dans Ball State University, Indiana, aux États-Unis 


\section{NAYAR LÓPEZ CASTELLANOS}

Politólogo y latinoamericanista, Profesor Investigador de la Facultad de Ciencias Políticas de la UNAM, Coordinador del Centro de Estudios Latino americanos de la FCPyS de la UNAM nayarlp[at]hotmail.com

\section{MATTHEW LORENZEN MARTINY}

Docteur en sociologie, Université Paris 1 Panthéon-Sorbonne Consultant dans le Consejo Nacional de Población (CONAPO), au Mexique 